\title{
Microstructure versus Size: Mechanical Properties of Electroplated Single Crystalline Cu Nanopillars
}

\author{
Andrew T. Jennings, ${ }^{1}$ Michael J. Burek, ${ }^{2}$ and Julia R. Greer ${ }^{1, *}$ \\ ${ }^{1}$ Engineering and Applied Sciences, California Institute of Technology, 1200 East California Boulevard, \\ Pasadena, California 91125, USA \\ ${ }^{2}$ University of Waterloo, 200 University Avenue West, Waterloo, ON N2L 3G1, Canada
}

(Received 18 January 2010; published 1 April 2010)

\begin{abstract}
We report results of uniaxial compression experiments on single-crystalline $\mathrm{Cu}$ nanopillars with nonzero initial dislocation densities produced without focused ion beam (FIB). Remarkably, we find the same power-law size-driven strengthening as FIB-fabricated face-centered cubic micropillars. TEM analysis reveals that initial dislocation density in our FIB-less pillars and those produced by FIB are on the order of $10^{14} \mathrm{~m}^{-2}$ suggesting that mechanical response of nanoscale crystals is a stronger function of initial microstructure than of size regardless of fabrication method.
\end{abstract}

DOI: 10.1103/PhysRevLett.104.135503

To date, one of the most common techniques for assessing mechanical properties of materials at the micron and submicron scales without imposing strong strain gradients is uniaxial deformation of micro and nanopillars [1-24]. The most prevalent method to fabricate pillars is by focused ion beam (FIB) [1-9,14,16-22,25-27]. Remarkably, at these length scales, face-centered cubic ( $\mathrm{fcc}$ ) metallic nanopillars exhibit a strong size-dependent strengthening: as the pillar diameter becomes smaller, its flow stress increases as a power law: $\sigma \propto d^{-n}$ where $d$ is the pillar diameter and $n$ lies between 0.5 and $0.7[1-7,11,23,26]$. Few alternate fabrication techniques, which do not rely on FIB, have also been used to produce nano- and micronsized samples. Interestingly, these samples show yield stresses near theoretical strength with no size effects, contrary to pillars produced by FIB [12,28,29]. Attainment of such high strengths has been attributed to the pristine initial microstructure in these pillars, i.e., no initial dislocations [28]. In contrast to any FIB-less technique, $\mathrm{Ga}^{+}$ion bombardment inevitably associated with FIB fabrication can introduce surface dislocation loops and precipitates, as well as instigate surface amorphization [14,30]. The presence of FIB-induced defects may have led several researchers to deduce that FIB-based fabrication methods may significantly contribute to strengthening. However, studies by Bei et al. [14] and by Lee et al. [23] showed that FIB machining of pristine or annealed Mo and $\mathrm{Au}$ pillars, in fact, reduces rather than increases their strengths to those significantly below the theoretical limit. Of note is recent work by Buzzi et al. [31] on embossed rather than FIB-machined submicron Ag pillars. While these authors also report a size effect, these $\mathrm{Ag}$ pillars are found to also deform through mechanical twinning, resulting in a more complex deformation mechanism than in other previously studied fec micropillars.

All of these studies clearly underline the importance of initial microstructure and its effect on the presence or absence of size effects. So far, FIB-less fabrication meth-
PACS numbers: 62.25. $-\mathrm{g}, 61.72 . \mathrm{Lk}, 61.80 .-\mathrm{x}, 61.82 . \mathrm{Bg}$

ods have only been able to produce features without dislocations, thereby rendering theoretical strengths, regardless of size, not surprising. Pillars fabricated by FIB, in contrast, contain as many as $10^{13}$ dislocations per $\mathrm{m}^{2}$ [32]. Therefore, in order to understand what drives the size effect, it is imperative to mechanically test pillars produced via FIB-less fabrication methods yet with nonzero dislocation densities. Recently, Burek et al. developed a FIBless method to produce single-crystalline $\mathrm{Cu}$ nanopillars by electroplating $\mathrm{Cu}$ into an array of electron beam patterned holes in a Poly(methyl methacrylate) matrix [33]. Thin films of electroplated copper have been reported to contain trace amounts $(.005 \%$ by weight $)$ of carbon from the plating process [34]. Since these contaminations are very small, if at all present, we believe that their effect on the attained nanopillar strengths is likely negligible. In this Letter, we show the results of uniaxial compression experiments performed on these $\sim\langle 111\rangle$ oriented, FIB-less $\mathrm{Cu}$ nanopillars with diameters between 100 and $500 \mathrm{~nm}$, with nonzero initial dislocation densities. We determine the pillar microstructure by performing site-specific TEM analysis on individual representative pillars, lifted out of the matrix and transferred onto TEM grid by using Omniprobe (Autoprobe 200, Omni Probe). Details of this procedure can be found in [35]. Figure 1(a) shows brightfield TEM image of a $100 \mathrm{~nm}$ pillar, with inset corresponding to diffraction pattern at [211] zone axis, which remained unaffected as the selected area diffraction aperture was moved along the pillar height, thereby demonstrating its single-crystalline nature. Analysis of the diffraction patterns' orientation with respect to pillar axes shows that the loading axis is $\sim\langle 111\rangle$. For example, the

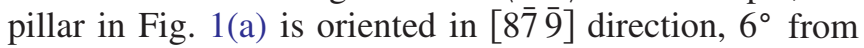
$[1 \overline{1} \overline{1}]$. This initial offset from the high-symmetry $\langle 111\rangle$ orientation causes rotation of the slip planes away from double slip condition [Fig. 1(b)], consistent with our observations that the slightly misaligned pillars deform by single slip. Those samples oriented perfectly along high- 


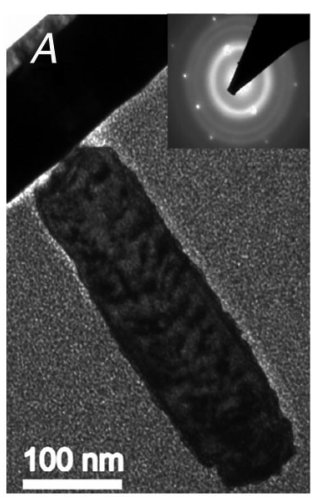

$B$

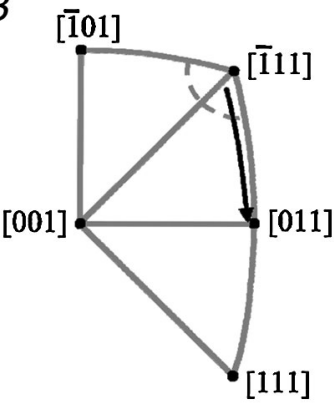

FIG. 1. (a) Bright-field TEM image of $\sim 100 \mathrm{~nm}$ diameter single-crystalline copper pillar. Inset diffraction pattern shows [211] zone axis. The rings in the diffraction pattern are from the partially nanocrystalline Pt deposition. (b) Stereographic triangle of [001] pole. Dashed line represents $10^{\circ}$ locus, the maximum deviation from the [111] loading axis. Arrow represents rotation of slip planes in compression away from the loading axis.

symmetry $\langle 111\rangle$ direction deform along multiple slip planes [Fig. 2(b)].

Pillars with diameters above $250 \mathrm{~nm}$ were compressed with a custom-made flat punch tip in Agilent G200 Nanoindenter. Pillars smaller than $250 \mathrm{~nm}$ were compressed in SEMentor, a custom-built in situ mechanical deformation instrument [5]. All compression tests were carried out at a nominally constant displacement rate of $2 \mathrm{~nm} / \mathrm{s}$ with simultaneous contact stiffness measurements via continuous stiffness measurement (CSM) option. Loaddisplacement data were converted into stress-strain by methodology outlined in [3]. Stiffness data were used to filter out tests that deviated from uniaxial behavior. Pillars were fabricated with aspect ratios of $\sim 3: 1$ to $6: 1$ in order to avoid any constraint hardening from the compression platens and to prevent buckling. A typical as-fabricated pillar morphology is provided in Fig. 2(a) and shows some surface roughness as well as the slightly imperfect pillarsubstrate interface. Figure 2(b) shows the same pillar after compression. Slip trace analysis shows that multiple slip systems were activated throughout the pillar volume indicating its likely $\langle 111\rangle$ orientation with respect to the loading direction.

Representative compressive true stress-strain curves corresponding to the deformation of pillars of four different nominal diameters are shown in Fig. 2(c). Each curve is characterized by nearly elastic initial loading followed by intermittent, discrete strain bursts. At the end of each strain burst, the pillar undergoes a short, nearly elastic loading until the stress is large enough to induce a new strain burst. This sporadic signature has been ubiquitously observed in the deformation of all nano and microscale pillars and is generally attributed to the highly stochastic nature of dislocation avalanches, and therefore, prevalent in sourcecontrolled plasticity $[1-11,13,14,16-19,36-39]$. Importantly, these electroplated pillars share the same flow behavior as those produced by FIB, clearly demonstrating
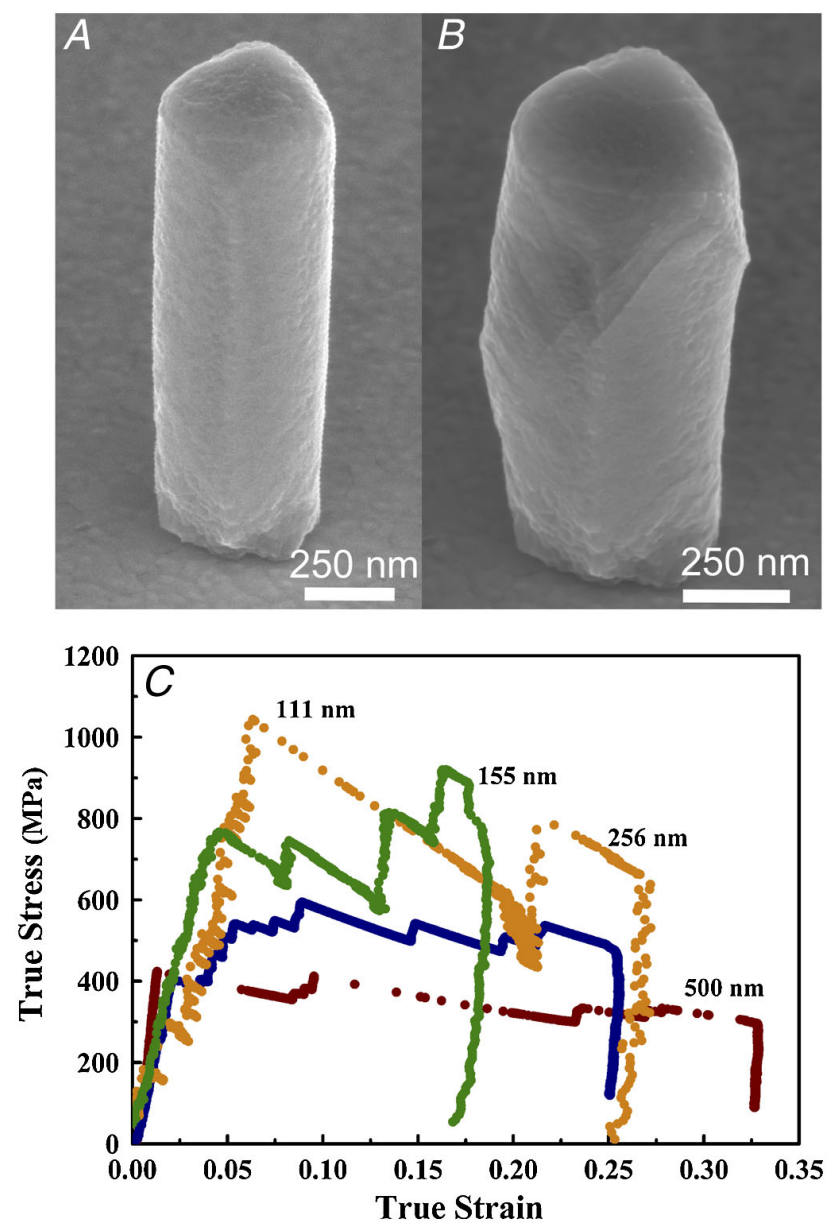

FIG. 2 (color online). SEM images of $\sim 500 \mathrm{~nm}$ diameter electroplated copper pillar (a) before and (b) after compression. Multiple symmetric slip lines can be seen in (b) corresponding to compression along $\langle 111\rangle$ loading axis. (c) Representative true stress vs strain curves. Numbers above each curve corresponds to pillar diameter. The plot clearly shows an increase in strength as a function of decreasing diameter.

that the same deformation mechanisms likely govern flow behavior of nanoscale samples regardless of fabrication methods. Furthermore, true stress-strain plots in Fig. 2(c) show unambiguous size effect: smaller pillars attain higher stresses compared to larger ones. The difference in initial loading slopes between the two smallest and two largest pillars is attributed to the different stiffnesses of the testing equipment (G200 vs SEMentor). In order to quantify the size-dependent strength, flow stresses at $10 \%$ strain of all compression tests plotted against pillar diameter on log-log scale are shown in Fig. 3. The compressive strengths of our nanopillars follow a power-law dependence with the slope of $-0.63 \pm .036$ (s. d.), a result strikingly similar to -0.6 , a value widely reported for various FCC metallic FIBfabricated pillars [11]. These results convincingly eliminate several of the previously conjectured main sources of size-dependent strength in FIB-fabricated nanoscale crystals, namely, precipitate strengthening $[30,40]$ and surface amorphization resulting in dislocation pileups and back 


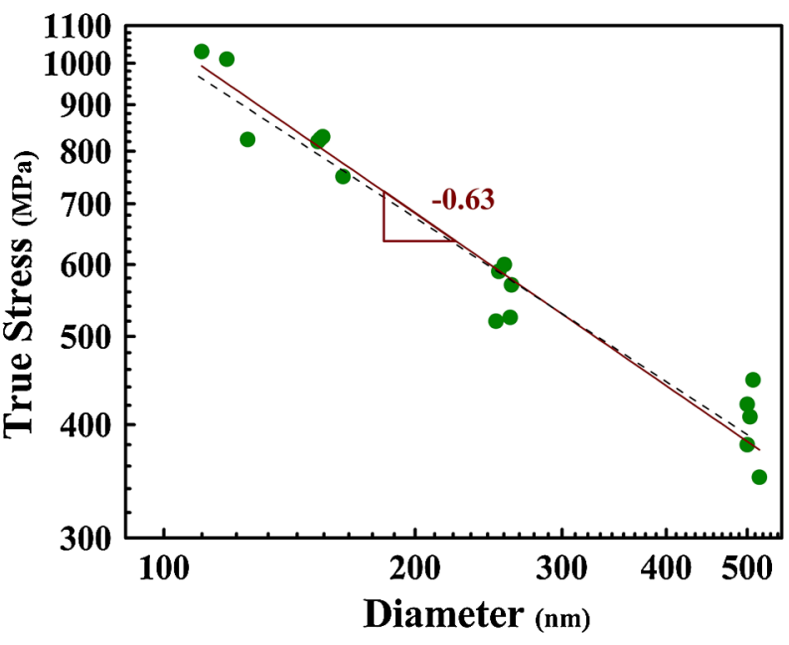

FIG. 3 (color online). Log-log plot of characteristic stresses plotted as function of pillar diameter. The data show a clear power-law trend (shown by dashed line) with $n=-0.63$, nearly identical to the trend demonstrated in FCC metals represented by the solid line [11].

stresses. Furthermore, the smallest $\mathrm{Cu}$ pillars with $100 \mathrm{~nm}$ diameters tested in this study reach only $\sim 15 \%$ of theoretical strength [41], a value significantly lower than that of other FIB-lessly fabricated pillars with zero initial dislocation densities [12,28,29], implying that our nanopillars contain initial mobile dislocations.

Comparing our results to those reported for FIBfabricated $\mathrm{Cu}$ samples, two key differences are apparent: our pillars attain lower flow stresses and negligible hardening relative to data in $[15,17,26]$. The authors of [17] demonstrate that both hardening and very high flow stresses attained by their pillars are due in part to the very stiff lateral support of their indenter system [15-17]. Furthermore, the low pillar aspect ratios used in their study led to the creation of dislocation pileups against the wider gripping supports during testing, causing additional hardening [24]. Rather, our compression results are consistent with the high aspect ratio tensile results reported by Kiener, et al. [16], as the longer sample geometry results in lowering the effective lateral stiffness of the entire system and thereby removing hardening effects due to dislocation pileups [15-17]. Also, the -0.63 slope reported here is larger than that reported by Kiener et al., -0.47 , also likely due to previously mentioned differences in lateral stiffness constraints.

In order to determine the initial dislocation density of our electroplated pillars, we performed site-specific weak beam dark field (WBDF) TEM analysis in FEI Tecnai TF20 (200 KeV). Samples were prepared in FIB by standard lift-out procedures utilizing Omniprobe (details can be found in [35]). Importantly, during thinning of the copper samples, only a negligible amount (if at all) of ion damage was introduced since the protective platinum layer remained on their surface. Figures 4(a) and 4(b) show WBDF images of an uncompressed $\mathrm{Cu}$ pillar, and the use of two depicted different diffraction conditions ensures we capture all dislocations within the pillar excluding only those with [110] Burgers vector. It is reasonable to expect that in $\mathrm{Cu}$, full lattice dislocations would dissociate into $\langle 121\rangle$-type partials with a corresponding stacking fault between them; therefore, all dislocations within the pillar should be visible in at least one of the two WBDF images. The estimated total dislocation line length in the boxed region is $317 \mathrm{~nm}$, as shown in Fig. 4(c). In order to compare this initial dislocation density to that of a FIB-machined pillar, Fig. 4(d) shows energy-filtered bright-field TEM images of a typical Au pillar produced in our lab, thinned down to electron transparency with a very low accelerating voltage of $1 \mathrm{kV}$ (FEI Hilsboro, OR). Unfortunately, this pillar was bent at the substrate interface in the process of thinning making WBDF, and therefore, Burgers vector analysis, unattainable. Dislocation densities were evaluated by dividing the total dislocation line length [Figs. 4(c) and 4(e)] by the volume in which they are contained. We find the initial dislocation density in our $\mathrm{Cu}$ nanopillars to be $1.5 \times 10^{14} \mathrm{~m}^{-2}$, i.e., the same order

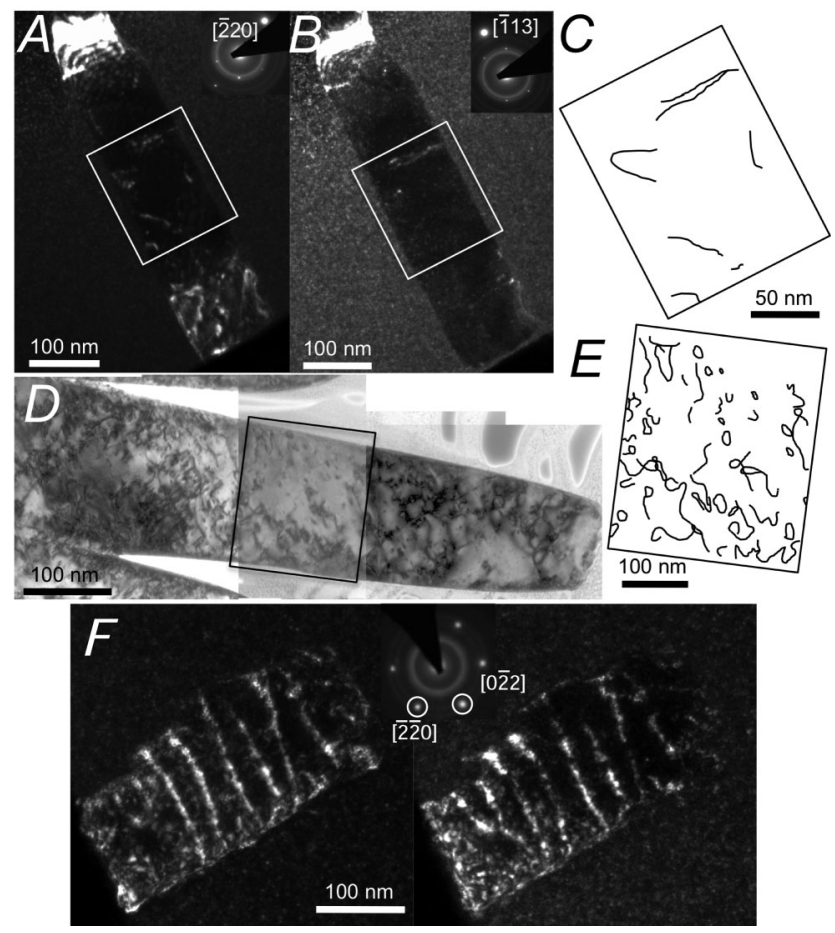

FIG. 4. (a), (b) WBDF images of $\sim 120 \mathrm{~nm}$ copper pillar. A layer of platinum was left covering the pillar such that the pillar did not endure any ion damage. Regions for dislocation density measurements are shown as the middle region where the deformation is expected to be homogeneous. (c) A zoomed-in rendition of the boxed region with outlined dislocation lines. (d) Bright-field energy-filtered TEM image of a $400 \mathrm{~nm}$ gold pillar fabricated with the FIB at $30 \mathrm{kV}$. This pillar was thinned down in successive steps to $1 \mathrm{kV}$ (FEI). (e) Enhanced view of dislocation density in the boxed region. (f) Dark field images of a compressed pillar illustrating that all of the remaining dislocations lie in the $\{111\}$ plane with no resolved shear stress. 
of magnitude as FIB-prepared Au nanopillars, $\rho=3 \times$ $10^{14} \mathrm{~m}^{-2}$. It is important to recognize that these represent an estimate of dislocation density rather than its exact value, as the dislocation length parallel to incident beam is not discernible, the precise distinction between pinned and mobile dislocations for the Au pillar is unavailable, and unambiguous identification of dislocation-based contrast features is challenging. While in bulk, a dislocation density of $10^{14} \mathrm{~m}^{-2}$ corresponds to a heavily cold-worked metal [42], the minimum attainable nonzero dislocation density in these small pillar volumes is $10^{12} \mathrm{~m}^{-2}$, equivalent to the existence of a single 7-atom loop in the entire pillar. A single dislocation line subtending the pillar diameter, for example, would increase the dislocation density by 2 orders of magnitude, i.e., corresponding to the value reported here. Figure 4(f) shows two dark field images of a compressed pillar, and, remarkably, demonstrates that all remaining dislocations lie in $\{111\}$ planes, which experience no resolved force during deformation, suggesting dislocation starvation as a dominant deformation mechanism [25]. Several atomistic and statistical simulations have corroborated this phenomenon in their explanations of size-dependent strengthening [36,43-47]. A recent review of these and other models can be found in Ref. [11].

In summary, our results demonstrate that $100-500 \mathrm{~nm}$ diameter single-crystalline $\mathrm{Cu}$ nanopillars created without the use of $\mathrm{Ga}^{+}$and containing several initial dislocations exhibit an identical size effect to the ones fabricated by FIB. This size effect manifests itself as "smaller is stronger" in a power-law fashion with exponent of $\sim-0.6$, consistent with most values reported to date for different FCC metals. This finding convincingly shows that plasticity at the submicron scale is truly a function of microstructure, which in turn defines size effect. At small scales, if the structure is initially pristine, i.e., without any dislocations, the material will yield and deform at close-totheoretical strengths, revealing no size effects. However, nanoscale crystals with nonzero initial dislocation densities display a remarkable dependence on size, as conveyed by many computational and experimental reports to date, and as demonstrated here-independently of fabrication technique.

We thank D. Jang for assistance in TEM operation and sample preparation and also B. Van Leer and L. A. Giannuzzi (FEI Company, Hillsboro, OR, USA) for assistance with TEM sample preparation. The authors gratefully acknowledge Kavli Nanoscience Institute at Caltech and financial support from the National Science Foundation through NSF CAREER Grant (DMR-0748267).

*To whom correspondence should be addressed: jrgreer@caltech.edu

[1] M. D. Uchic et al., Science 305, 986 (2004).
[2] D. M. Dimiduk, M.D. Uchic, and T.A. Parthasarathy, Acta Mater. 53, 4065 (2005).

[3] J. R. Greer, W. C. Oliver, and W. D. Nix, Acta Mater. 53, 1821 (2005).

[4] C. P. Frick et al., Mater. Sci. Eng. A 489, 319 (2008).

[5] J. Y. Kim and J. R. Greer, Acta Mater. 57, 5245 (2009).

[6] C. A. Volkert and E. T. Lilleodden, Philos. Mag. 86, 5567 (2006).

[7] K. S. Ng and A. H. W. Ngan, Acta Mater. 56, 1712 (2008).

[8] S. Brinckmann, J.-Y. Kim, and J. R. Greer, Phys. Rev. Lett. 100, 155502 (2008).

[9] Z.W. Shan et al., Nature Mater. 7, 115 (2008).

[10] S. H. Oh et al., Nature Mater. 8, 95 (2009).

[11] M. D. Uchic, P. A. Shade, and D. M. Dimiduk, Annu. Rev. Mater. Res. 39, 361 (2009).

[12] H. Bei et al., Scr. Mater. 57, 397 (2007).

[13] H. Bei et al., Acta Mater. 56, 4762 (2008).

[14] H. Bei et al., Appl. Phys. Lett. 91, 111915 (2007).

[15] D. Kiener, W. Grosinger, and G. Dehm, Scr. Mater. 60, 148 (2009).

[16] D. Kiener et al., Acta Mater. 56, 580 (2008).

[17] D. Kiener, C. Motz, and G. Dehm, Mater. Sci. Eng. A 505, 79 (2009).

[18] J.-Y. Kim and J. R. Greer, Appl. Phys. Lett. 93, 101916 (2008).

[19] J.-Y. Kim, D. Jang, and J.R. Greer, Scr. Mater. 61, 300 (2009).

[20] E. M. Nadgorny, D. M. Dimiduk, and M.D. Uchic, J. Mater. Res. 23, 2829 (2008).

[21] B. Moser et al., J. Mater. Res. 22, 1004 (2007).

[22] F. Ostlund et al., Adv. Funct. Mater. 19, 2439 (2009).

[23] S. W. Lee, S. M. Han, and W. D. Nix, Acta Mater. 57, 4404 (2009).

[24] D. Kiener, C. Motz, and G. Dehm, J. Mater. Sci. 43, 2503 (2008).

[25] J. R. Greer and W. D. Nix, Phys. Rev. B 73, 245410 (2006).

[26] D. Kiener et al., Adv. Eng. Mater. 8, 1119 (2006).

[27] C. A. Volkert and A. M. Minor, MRS Bull. 32, 389 (2007).

[28] S. S. Brenner, J. Appl. Phys. 27, 1484 (1956).

[29] G. Richter et al., Nano Lett. 9, 3048 (2009).

[30] D. Kiener et al., Mater. Sci. Eng. A 459, 262 (2007).

[31] S. Buzzi et al., Philos. Mag. 89, 869 (2009).

[32] D. M. Norfleet et al., Acta Mater. 56, 2988 (2008).

[33] M. J. Burek and J. R. Greer, Nano Lett. 10, 69 (2010).

[34] M. Stangl et al., Microelectron. Eng. 82, 189 (2005).

[35] T. M. Moore, Microscopy Today 13, 40 (2005).

[36] D. M. Dimiduk et al., Science 312, 1188 (2006).

[37] J. R. Greer, C. R. Weinberger, and W. Cai, Mater. Sci. Eng. A 493, 21 (2008).

[38] P. A. Shade et al., Acta Mater. 57, 4580 (2009).

[39] F. F. Csikor et al., Science 318, 251 (2007).

[40] J. A. El-Awady et al., Phys. Rev. B 80, 104104 (2009).

[41] S. Ogata et al., Phys. Rev. B 70, 104104 (2004).

[42] D. Hull and D. J. Bacon, Introduction to Dislocations (Elsevier Ltd., Oxford, 2001).

[43] J. A. El-Awady, M. Wen, and N. M. Ghoniem, J. Mech. Phys. Solids 57, 32 (2009).

[44] S. I. Rao et al., Acta Mater. 56, 3245 (2008).

[45] K. S. Ng and A. H. W. Ngan, Philos. Mag. 88, 677 (2008).

[46] C.R. Weinberger and W. Cai, Proc. Natl. Acad. Sci. U.S.A. 105, 14304 (2008).

[47] T. A. Parthasarathy et al., Scr. Mater. 56, 313 (2007). 